\title{
Applications of Advanced Shielded Planar EM Analysis Techniques to Antenna Analysis
}

\author{
J.C. Rautio \\ Sonnet Software,U.S.A., rautio@ sonnet software. com, fax: +1.315.451.1694
}

Keywords: Antenna Analysis, Method of Moments, Model Extraction, RFID.

\begin{abstract}
With appropriate setup, shielded planar EM (electromagnetic) analysis can be used for antenna analysis. While shielded EM analysis can not handle the variety of antenna problems that unshielded analysis can, shielded analysis offers extreme numerical robustness. This robustness allows, in turn, rigorous identification, quantification, and elimination of analysis error sources. This robustness also allows perfectly calibrated groups of internal ports, unique to shielded EM analysis. This in turn leads to new efficient design methodologies and also allows a new compact model extraction (actually, synthesis) capability. These capabilities are described and illustrated with examples.
\end{abstract}

\section{Introduction}

The next section provides an overview of a shielded, planar, method of moments analysis as applied in this paper. The overview is conceptual; it uses no equations and little background in EM theory is needed. Next, we describe the relative advantages and disadvantages of shielded versus unshielded planar EM analysis. The concept of "perfect" port calibration is then introduced. This capability is critical for rigorous analysis error attribution. This is followed by a description of how shielded EM analysis can be applied to antennas, Figure 1. A validation example of a dipole in free space is presented using the perfect port calibration. The dipole example is also used to illustrate parameterized sweeps, followed by an illustration of a new compact model synthesis capability that is critically dependent on perfect port calibration. This example is continued with a broad band 900 $\mathrm{MHz}$ dipole, and a multiple patch antenna. The manner in which perfectly calibrated ports are used to include circuit theory networks (S-parameters, or lumped networks) within antennas is illustrated with the broad band dipole. This is useful for loading antennas and for including the effect of feed networks.

\section{Shielded EM Analysis Overview}

The full theory used in this paper is described in [1] and [2]. The software is commercially available [3]. Here, we present a conceptual description only. Minimal knowledge of EM theory is needed.

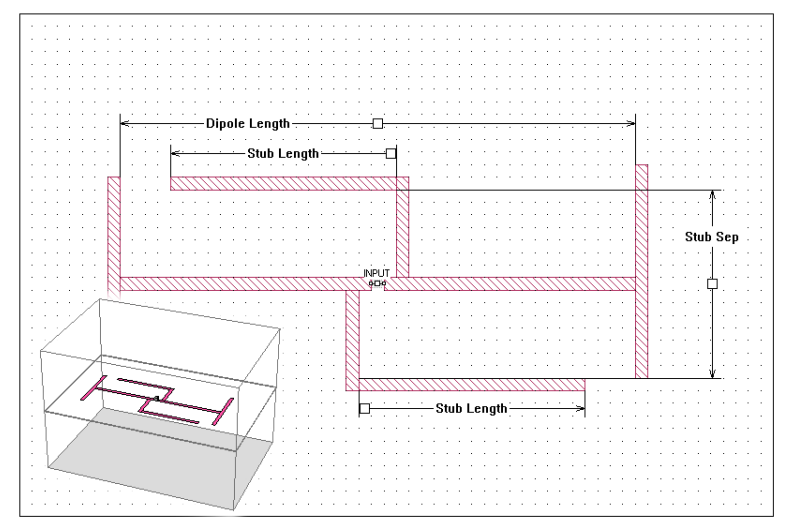

Figure 1: An antenna in a shielded analysis. The top and bottom covers are set to 377 Ohms per square. The perfectly conducting box sidewalls are moved about 10 times further away from the antenna than shown.

In this Method of Moments (MoM) approach, we divide the metal of a multi-layer circuit into small subsections. Only the metal of the circuit is meshed; the volume of the circuit is not meshed.

Next, we numerically calculate the voltage induced on one subsection due to current that has been placed on another subsection. The subsection with current can be viewed like a tiny transmitting antenna. The subsection on which voltage is induced can be viewed like a tiny receiving antenna. We repeat this pair-wise coupling calculation for all possible pairs of subsections. For $\mathrm{N}$ subsections, this fills an NxN matrix (the "moment matrix"). To solve for the current distribution we invert this matrix. Once the current distribution is known, evaluation of things like the antenna pattern, input impedances, etc., follows immediately.

The central problem is calculating the voltage induced on one subsection due to current on another subsection. This is where the mathematics can become difficult. Specifically, the Green's function (i.e., the fields due to an infinitesimal dipole) must be integrated four times. The Green's function must first be integrated over the two dimensions of the subsection with current on it (i.e., the source subsection). This yields the fields everywhere due to the patch of current. This result must then be integrated two more times over the area of the subsection on which we are calculating the voltage (i.e., the field subsection). While this concept is simple, the actual equations can be intimidating. 
This paper was presented at the European Antennas and Propagation Conference (EuCAP2007) in Edinburgh Scotland on 15

Nov 2007 and is copyrighted by the IET. A correction to the published paper is noted at the end of this Sonnet distributed

copy.

For the analysis of planar multi-layered circuits contained in a rectangular shielding box, the Green's function is a sum of rectangular waveguide modes, the sidewalls of the shielding box form the sidewalls of the waveguide tube, extending in the vertical direction. Thus the Green's function is a weighted sum of sines and cosines. Once the equation for this sum is determined, integration is easy. Sine goes to cosine and cosine goes to sine. Surprisingly, after performing all four integrations analytically, the form of the sum is exactly the same. The only thing that has changed is the weighting coefficient for each term in the sum.

All the integration for shielded EM analysis was performed analytically three decades ago using pencil and paper. No numerical integration is ever used anywhere in the analysis. In filling the moment matrix, all we do is perform a weighted sum of sines and cosines. In fact, this sum is performed quickly and efficiently using an FFT (Fast Fourier Transform). It is this characteristic that provides both the advantages and disadvantages of shielded EM analysis.

\section{Shielded Versus Unshielded}

First, a style note: We use the neutral terms "shielded" and "unshielded". The terms "closed" and "open" suggest a predisposition as to which one is better.

An unshielded planar EM analysis proceeds very much as described for shielded analysis above. The main difference is that the Green's function is a Sommerfeld integral. As with the FFT used for shielded analysis, this is both an advantage and a disadvantage.

An advantage of the unshielded approach is that it automatically includes radiation with no special setup. Another advantage extends from the fact the four dimensional integration of the Sommerfeld integral must be performed numerically. This takes the form of an advantage when one realizes that the numerical integration can be performed over any desired area. Subsections can be nearly any shape, size, and orientation. This allows analysis of a wide variety of antennas.

The mating disadvantage is that numerical integration can be slow and it necessarily entails numerical precision error, especially for the rapidly varying Sommerfeld integral. In unshielded tools, the numerical integration is typically carried out to three decimal digits of precision. This yields a nominal $60 \mathrm{~dB}$ numerical noise floor provided subsection aspect ratio is not too extreme, subsection size is not too small, and moment matrix size is not too large. This is good enough for some applications, but it is not good enough for all.

The disadvantage for shielded analysis extends from the FFT used to fill the moment matrix. Recall that in signal processing, you must first uniformly sample the signal. For shielded MoM, the 2-D FFT is taken over the area of the circuit substrate. Thus the surface of the substrate is meshed based on a uniform underlying FFT mesh. The mesh can easily be, say, 1000 cells by 1000 cells (2-D FFT time is about one second, no more than three FFT's are needed for one level of circuitry). This FFT mesh means that curving lines, for example, must have a fine stair case imposed on their edges.

But the FFT also embodies the shielded approach's strength: The moment matrix is filled to full numerical precision. This means that the analysis noise floor is often well below $100 \mathrm{~dB}$ down, subsection aspect ratio can become extreme, lots of very tiny subsections are no problem, and when the moment matrix becomes large there is no impact on noise floor (provided a good pivoting is used).

These advantages of a shielded analysis provide its extreme robustness and allow detailed investigation of very small analysis error sources as well as enabling various exceptionally efficient design methodologies.

In applied work, the skilled designer will have both a shielded and an unshielded tool available, and will be familiar with their relative advantages and disadvantages.

\section{Perfect Port Calibration}

The dominant application for shielded EM analysis is microwave circuits where accuracy is a major concern. The two main error sources for most EM analyses are error due to subsection size and error due to imperfect port calibration.

For a robust EM analysis, as we have with shielded EM analysis, error due to subsection size decreases (usually linearly) with decreasing subsection size. Because subsection size can be taken very small without numerical difficulty, this is a valuable approach to determining analysis error. Simply cut the cell size in half and, usually, the error cuts in half. If you have any doubt, cut the cell size in half again, and see if the error is still decreasing by about half. Using this approach, analysis error can, with little effort, be reduced to less than $1 \%$ quantified, and with care to under $0.05 \%$ quantified.

An EM circuit or antenna is excited with various kinds of circuit theory ports. All such ports introduce one or another kind of error. For example, the gap port excitation used in our shielded EM analysis typically introduces a small shunt capacitance. Using techniques analogous to network analyzer calibration [4] and [5], we can perfectly characterize that shunt capacitance and perfectly remove it, to within numerical precision. This includes multiple closely coupled ports and assumes that no port connecting lines are over moded. However, as presented in [4] and [5], this perfect calibration is available only for ports on the outside edges of a circuit, along the box wall.

Recently, I developed a technique to extend this perfect port calibration to multiple coupled internal ports [6]. Detailed validation (by means of extreme "torture tests") is described in [7]. Oddly, it seems I am the only researcher with major commercial application that publishes the port calibration 
This paper was presented at the European Antennas and Propagation Conference (EuCAP2007) in Edinburgh Scotland on 15

Nov 2007 and is copyrighted by the IET. A correction to the published paper is noted at the end of this Sonnet distributed copy.

routines being used. Just think of where we would be today if network analyzer calibration algorithms had been kept secret!

Quickly summarizing the impact of perfectly calibrated internal ports on, say, RFIC design: One can replace all resistors, capacitors, transistors, etc. in an RFIC layout with perfectly calibrated ports and then EM analyze the remaining interconnect. Then using circuit theory the RFIC can be populated with models or S-parameters for the removed components. The design is quickly tuned for optimum performance with each candidate design analyzed at circuit theory speed. Hundreds of potential designs can be considered with full EM accuracy in a single morning. In the old way of doing things, each candidate design must be subjected to full EM analysis. This way is no longer competitive.

Perfect port calibration requires a perfect short circuit calibration standard. For shielded EM analysis, this is available in the perfectly conducting sidewalls of the shielding box. In order to do a similar port calibration in an unshielded analysis, images must be used to create approximate short circuit calibration standards. In addition, radiation can not be allowed during the calibration procedure. These factors, combined with the limited numerical precision of unshielded analysis, effectively preclude practical application of this calibration in an unshielded analysis. The impact of perfectly calibrated ports on antenna design is illustrated in examples below.

\section{Problem Setup for Radiation}

In a shielded EM analysis, the sidewalls of the box form a rectangular tube waveguide extending vertically. The fields inside the box are represented as a weighted sum of rectangular waveguide modes. The top and bottom covers of the box terminate each end of the waveguide tube. In performing a shielded analysis, we can set the impedance of the top and bottom covers to any value we want. To analyze a radiating circuit, we first move the sidewalls far enough from the circuit so that they do not restrict radiation. Then we move the top and bottom covers out of the near field of the radiator and set the cover impedances to $377 \mathrm{Ohms}$ per square, the impedance of free space.

Moving the box walls far from the radiator increases the size of the FFT (remember, the FFT is taken over the area of the substrate). However, as long as most of the substrate has little additional circuit metal, the moment matrix size is left unchanged and the increased FFT typically has little impact. So a large substrate area can be easily analyzed.

The thickness (or thin-ness) of a dielectric layer is likewise no problem. The thickness of a dielectric layer impacts only the argument of a tangent function, there is no impact on speed or accuracy. In addition, substrate loss and conductivity simply make the tangent function complex. There is no impact on accuracy. Since evaluation of the tangent function is inconsequential, substrate loss has no impact on speed.

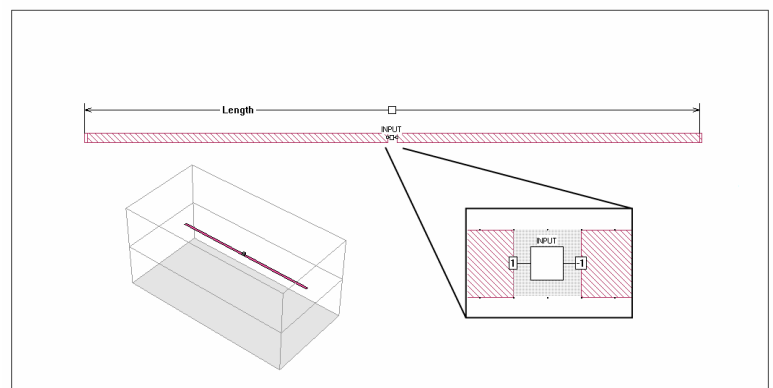

Figure 2: A dipole is used to evaluate the error introduced by the presence of the conducting sidewalls (actual sidewalls are about 10 times further away than shown).

So, how far must the covers and sidewalls be moved away from the antenna? We determine this by numerical experiment. We try one distance, then double that distance and see how much difference there is.

\section{Dipole Example}

To illustrate, Figure 2 shows a half-wave dipole in free space (i.e., Erel = 1) in a box. For a correct analysis, the input impedance at resonance is $73.1 \mathrm{Ohms}$. In this figure, the box sidewalls are much too close to realize this. It is shown this way so you can see both the box and the details of the dipole.

The dipole is $13 \mathrm{~cm}$ long and the actual box size is $160 \mathrm{~cm}$ on a side (over ten times bigger than illustrated). The top and bottom covers are $15 \mathrm{~cm}$ (about one half wavelength) from the dipole. The dipole is not a wire; rather it is a flat strip 0.2 $\mathrm{cm}$ wide. The port region is a perfectly calibrated $0.2 \mathrm{~cm}$ square. Actual antenna input impedance can depend strongly on the physical details of the feed point region. Because we use perfectly calibrated ports here, all EM effects of the port are exactly removed. If a separate model for the specific feed point being used is available, it can then be inserted into the perfectly calibrated ports using circuit theory. For this example, we present the results without any additional feed structure model inserted. What is nice about this for validation is that we know the exact answer at resonance.

As mentioned above, radiation is not allowed during perfect port calibration. This of course means the area covered by the calibrated port group must be small (as it is here, $0.2 \mathrm{~cm}$ square), but it also means that the calibration standards analyzed in the course of calibration must be small and nonradiating. In this case, we specified a "calibration standard length" of $1 \mathrm{~cm}$, which means the complete calibration standard is $2.2 \mathrm{~cm}$ in length and is contained between shielding sidewalls. Thus, there is no radiation during the calibration procedure. In addition, as an option the user may choose a floating local ground reference for the calibrated ports, or a global ground reference. Because the global ground is unavailable, we select a floating local ground.

Figure 3 shows the reflection coefficient (S11) for the dipole. The reflection coefficient at resonance corresponds to 71.43 
This paper was presented at the European Antennas and Propagation Conference (EuCAP2007) in Edinburgh Scotland on 15 Nov 2007 and is copyrighted by the IET. A correction to the published paper is noted at the end of this Sonnet distributed copy.

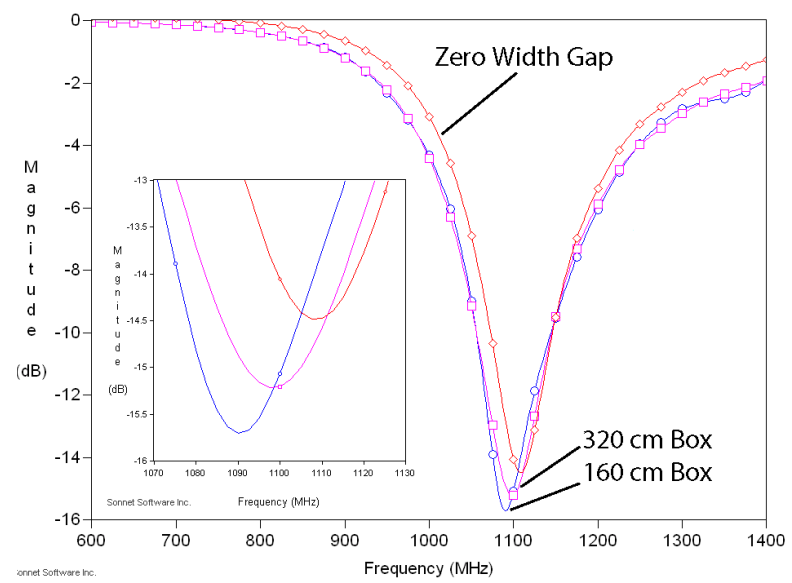

Figure 3: Moving the sidewalls farther away $(320 \mathrm{~cm}$ box $)$ moves the dipole closer to 73.1 Ohms $(-14.53 \mathrm{~dB}$ S11) input impedance. Going from a $0.2 \mathrm{~cm}$ port gap to zero port gap yields $73.5 \mathrm{Ohms}$ input impedance.

Ohms, about 1.6 Ohms below the correct answer. How much of this $1.6 \mathrm{Ohms}$ is due to the presence of box sidewalls? We doubled the box size laterally to $320 \mathrm{~cm}$ on a side (top and bottom covers left at $15 \mathrm{~cm}$ from the dipole). The result is also plotted in Figure 3. The input impedance is now 71.65 Ohms, $5 \mathrm{MHz}$ higher. The difference of $0.2 \mathrm{Ohms}$ and $5 \mathrm{MHz}$ is due to the box sidewalls. Thus only a small portion of the total $1.6 \mathrm{Ohm}$ error can be justified by the presence of the box sidewalls at their present location.

For the remaining 1.4 Ohms error, we explored increasing the box size further, moving the top and bottom covers further away, and decreasing the width of the dipole strip. The only significant change was decreasing the dipole strip width to 0.1 $\mathrm{cm}$, and that actually increased the error, decreasing the input impedance $0.4 \mathrm{Ohms}$. Next, leaving the line width at $0.1 \mathrm{~cm}$, we changed the input port region from $0.2 \mathrm{~cm}$ long (the size of the anticipated feed structure) to a width of zero (a single infinitesimal gap). The input impedance moved to $73.5 \mathrm{Ohms}$, $0.4 \mathrm{Ohms}$ too high. Further convergence analysis on line width should eliminate most of this remaining error.

Notice that in the above, I made no statement that the agreement is "good". That judgement can only be made by a designer who has specific requirements to meet. The only thing I can do as an objective researcher is to provide an estimate of the error so that that judgement can be made by one who also has a set of requirements. Lacking information on the user's requirements, it is improper to make any overall judgement as to "good" or "bad".

Another error source that we investigated is error due to interpolation. The default interpolation requires analysis at only four frequencies for this dipole and then it interpolates the entire $600-1400 \mathrm{MHz}$ result. However, this results in maximum interpolation error of about $2 \mathrm{Ohms}$ at resonance. By forcing the interpolation to over sample (indicated by the data point markers), we reduce the maximum interpolation error to 0.03 Ohms. This is determined by running a second

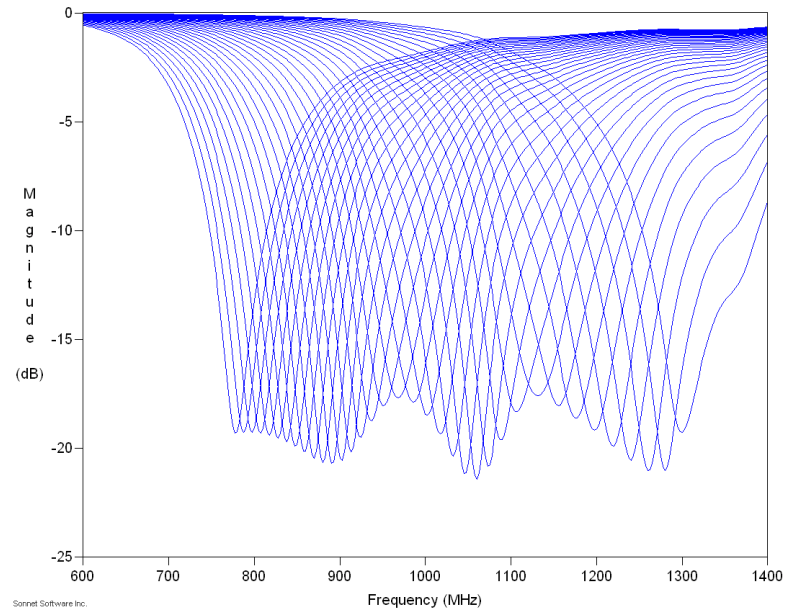

Figure 4: Sweeping the dipole length from $10 \mathrm{~cm}$ to $17 \mathrm{~cm}$ with a step of $0.2 \mathrm{~cm}$ shows that the minimum $\mathrm{S} 11$ varies a few $\mathrm{dB}$. This is due to interaction with the box sidewalls.

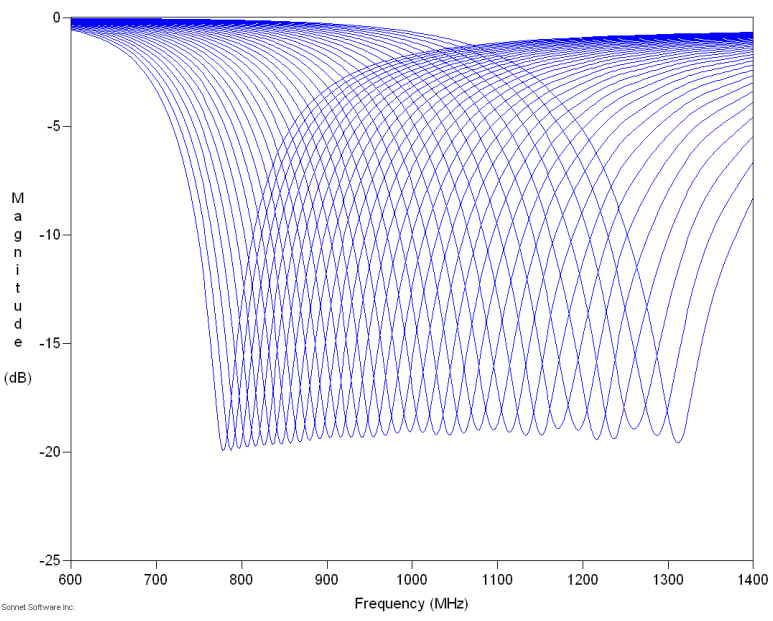

Figure 5: With the box size doubled to $320 \mathrm{~cm}$ square, the interaction with the sidewalls is almost completely gone.

analysis, without interpolation, at interpolated frequencies. We deem $0.03 \mathrm{Ohm}$ interpolation error to be adequate for our present needs. The same over sampling is used in subsequent dipole analyses.

\section{Parameterized Dipole Example}

For this case, we modify the free space dipole by adding a thin substrate $0.05 \mathrm{~cm}$ thick with Erel $=3.0$. The intended application is RFID at $900 \mathrm{MHz}$. Figure 2 shows the "Length" parameter, which we swept from 10 to $17 \mathrm{~cm}$ in steps of $0.2 \mathrm{~cm}$. The resulting reflection coefficient for each length is in Figure 4. Note that the minimum S11 varies as a function of length. Why does this happen?

One hypothesis is that this is due to the box sidewalls. However, in the free space dipole example, above, we saw that the box sidewalls had only about $0.2 \mathrm{Ohms}$ of effect on the resonant input impedance. The variation in reflection 
This paper was presented at the European Antennas and Propagation Conference (EuCAP2007) in Edinburgh Scotland on 15

Nov 2007 and is copyrighted by the IET. A correction to the published paper is noted at the end of this Sonnet distributed copy.

coefficient of Figure 4 corresponds to about 6 Ohms (59.7 to 65.7 Ohms). How could this be due to the box sidewalls?

Let's extend the hypothesis. Perhaps the thin dielectric layer not only lowers the resonant input impedance, but it also allows a surface wave mode that brings the sidewalls into interaction with the dipole. We can easily test this hypothesis numerically. Double the size of the box (laterally) and see what happens, Figure 5.

The ripple in minimum reflection coefficient is now much faster and the amplitude is significantly reduced, substantially supporting our hypothesis.

Up to now, we have been treating the box sidewalls as an error source. However, this is not always the case. Sometimes, there might actually be box sidewalls interacting with the antenna in the form of a recessed cavity used for mounting the antenna. In other cases, the environment at some distance from the antenna might not be controlled. Anything could be there. With the results of Figures 4 and 5, we have a reasonable idea of the effect of random foreign objects being introduced. Of course, if you need knowledge of interaction with a specific foreign object, a 3-D volume meshing EM analysis should be invoked.

This also brings us to a weakness of unshielded EM analysis. Unshielded tools assume the substrate goes to infinity. Any surface wave that is launched is never reflected. The corresponding weakness for shielded tools is that $100 \%$ of any surface wave is reflected at the box sidewalls. The typical situation is between the two extremes. Thus, analysis with both shielded and unshielded codes bounds the problem.

\section{Dipole Compact Model Synthesis}

My personal research for the last several years has been compact model synthesis using EM analysis data [8], patent pending. The technique needs only precise EM analysis data for input. It does not need geometric knowledge about the component being modelled, nor does it need a guess as to a potential lumped model. However, the approach is extremely sensitive to even the smallest non-physical error in input data. Thus, measured data and data from anything other than an exactly calibrated shielded analysis is unusable.

Primary application was assumed to be microwave circuits. Since the output is a compact lumped model, the approach was not expected to succeed for antennas. However, as we see in Figure 6, a simple broad band model exists for the dipole. This lumped model is derived from the Length $=15 \mathrm{~cm}$ dipole of the previous section. Similar models for numerous other dipoles have also been synthesized. The implications of this most unexpected result have not yet been explored.

\section{Broad Band Dipole}

The typical near field RFID environment is uncontrolled. There might be a bag of oranges, a case of soda, or the side of

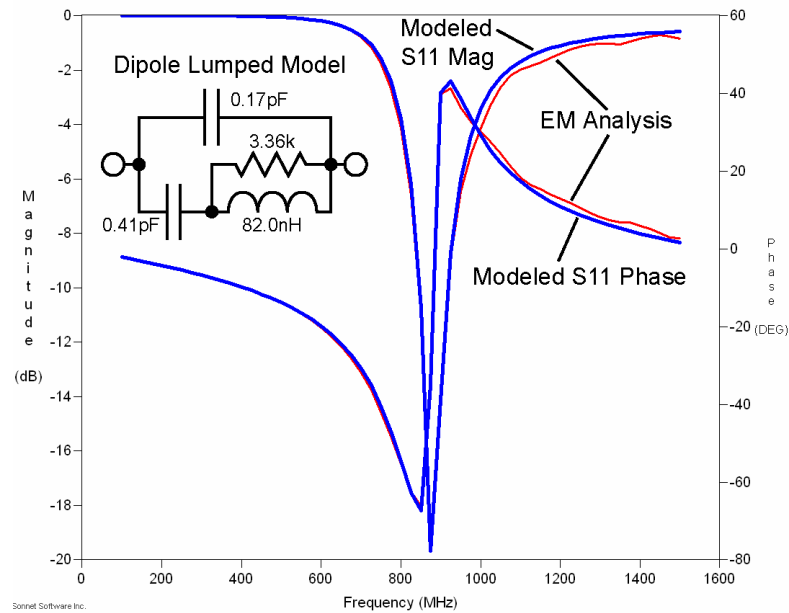

Figure 6: An unexpected result, we are able to use a new compact model synthesis technique to extract a broad band lumped model for a dipole.

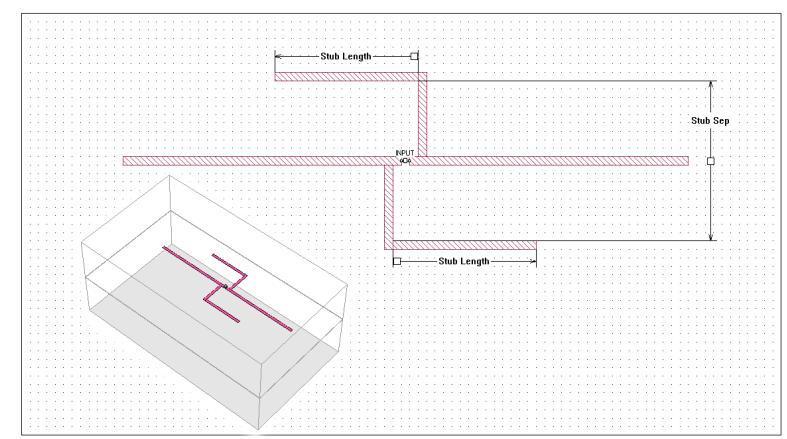

Figure 7: This geometry for a broad band dipole is similar to two element log periodic antenna.

a shopping cart nearby. All these things modify the dipole resonant frequency and impedance. Thus we would like to design a dipole having wide bandwidth. A typical dipole has only a few percent bandwidth. Since the EM analysis time for the dipole discussed above is just several seconds per frequency, it is possible to try many experiments in a short time. So I sat down in my comfy chair one Saturday afternoon and after an hour or so of experiments, I hit on the geometry of Figure 7. I call this the quasi-log periodic antenna [9]. It is like two elements of a log-periodic antenna.

But what values to use for the indicated dimensions? Again, this is a really fast analysis, so I just ran a parameterized sweep, Figure 8. There are 55 frequency sweeps, 294 frequencies per sweep; total time was 24 minutes on a notebook computer. The best result is highlighted. The indicated dimensions provide about $300 \mathrm{MHz}$ of bandwidth centered on $1100 \mathrm{MHz}$ into $50 \mathrm{Ohms}$. Since foreign objects in the near field tend to lower the resonant frequency, the desired $950 \mathrm{MHz}$ is the low end of the bandwidth. There is sufficient bandwidth in this dipole to host all of the different $900 \mathrm{MHz}$ worldwide bands.

To illustrate using the perfectly calibrated internal ports, assume that it is desired to load the main dipole with surface 
This paper was presented at the European Antennas and Propagation Conference (EuCAP2007) in Edinburgh Scotland on 15 Nov 2007 and is copyrighted by the IET. A correction to the published paper is noted at the end of this Sonnet distributed copy.

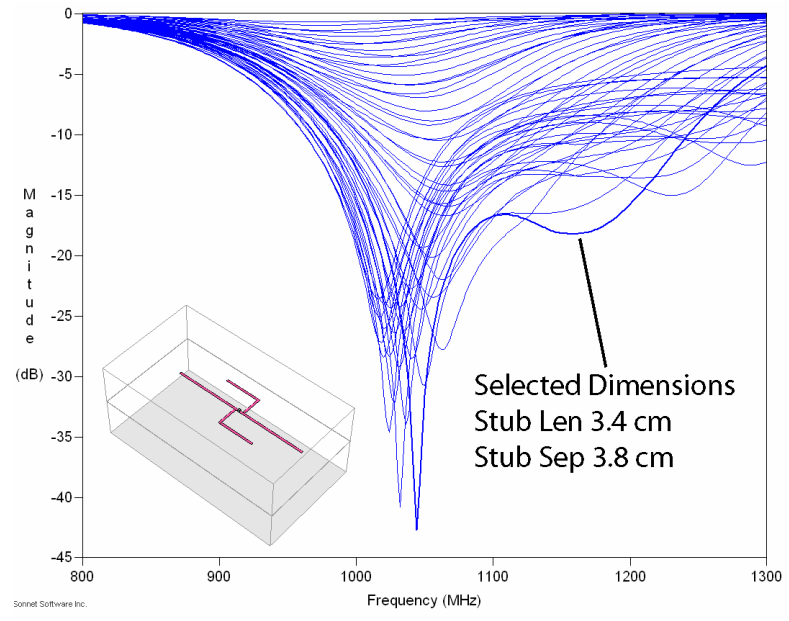

Figure 8: A parameter sweep through stub length and stub separation yields one result with very wide bandwidth.

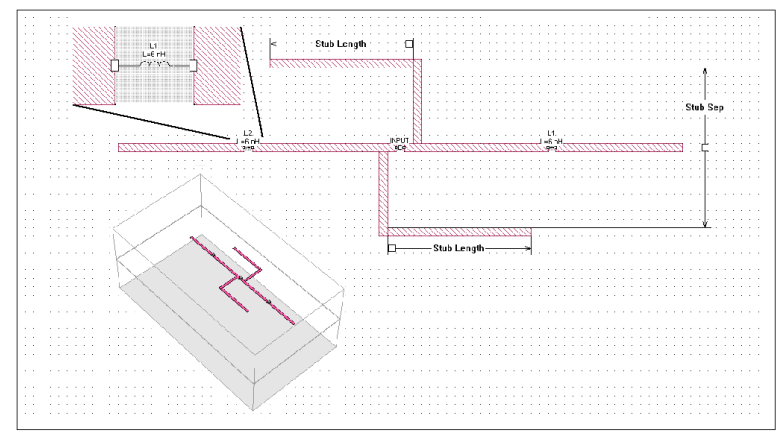

Figure 9: Using perfectly calibrated internal ports to insert inductive loading into the dipole arms, illustrated here with ideal inductors. Surface mount inductor models or measured data can be used too.

mount inductors to make it resonate at a lower frequency, Figure 9. For initial analysis, we use ideal circuit theory inductors as illustrated. After an initial full EM analysis, the inductors can be changed and the original EM analysis is used each time. The new results are available essentially instantly. While inserting lumped elements into an MoM analysis is nothing new, we can now do it with perfectly calibrated ports. This is important for high accuracy requirements, especially at high frequency.

With a choice of $6 \mathrm{nH}$ loading inductors, the response is shown in Figure 10. A model or S-parameters for a specific surface mount inductor can be inserted as well.

\section{Multiple Patch Antenna}

One of the classic antennas we have used for many years to illustrate antenna analysis is the triple patch antenna [10]. Patch antennas have reduced radiation horizontally; most of the radiation is vertically, perpendicular to the plane of the patch. This means that we can perform the analysis with the box sidewalls closer to the antenna. In fact, entirely adequate analysis usually results with the box sidewalls one half wavelength from the edge of the patch. The middle patch is probe fed from below on the right side, Figure 11.

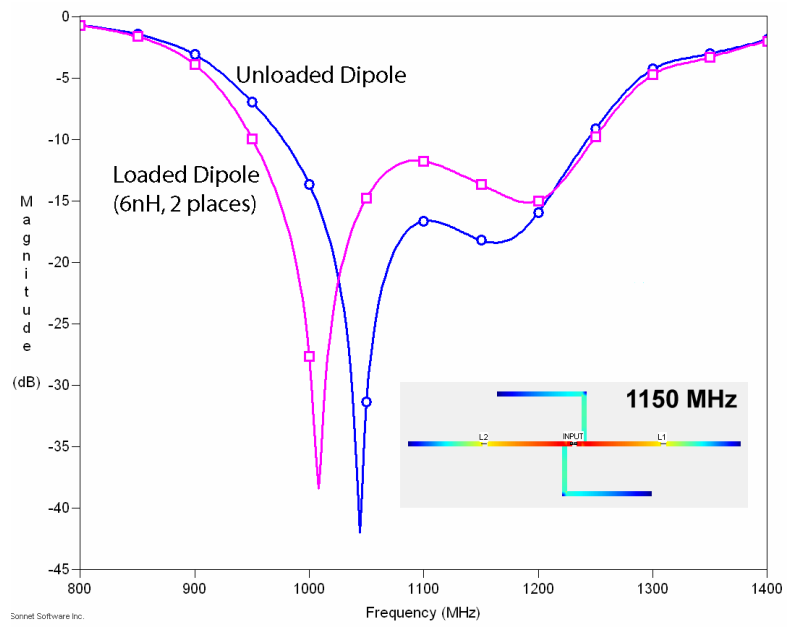

Figure 10: Loading inductors are inserted into the dipole by means of perfectly calibrated ports, lowering the low band edge. Note the current distribution includes the effect of the inductors, L1 and L2.

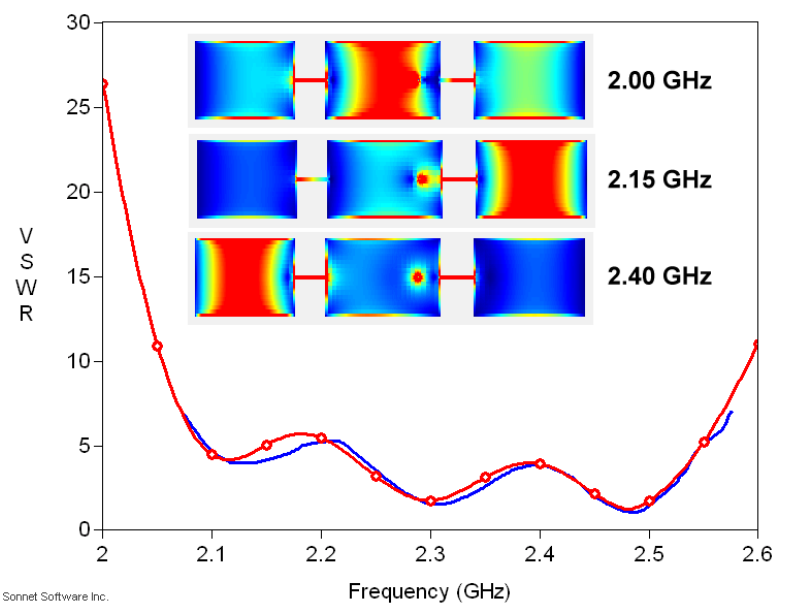

Figure 11: Measured (no data markers) versus calculated (circle data markers) VSWR for the triple patch antenna.

\section{Conclusion}

We have described how a shielded planar EM analysis is used for antenna analysis. A conceptual description of the method of moments technique used and a trade-off of shielded versus unshielded EM analysis is included. An advantage of shielded analysis is numerical robustness and the ability to precisely quantify error sources. A dipole and a broad band dipole are investigated to demonstrate this capability. Errors are quantified to tenth $\mathrm{Ohm}$ precision and causality assigned to their sources. This investigation is facilitated by the use of perfectly calibrated ports, which can be achieved in shielded EM analysis. The perfectly calibrated ports are also used to insert circuit theory elements (like feed structures or lumped loading circuits) into antennas. 
This paper was presented at the European Antennas and Propagation Conference (EuCAP2007) in Edinburgh Scotland on 15

Nov 2007 and is copyrighted by the IET. A correction to the published paper is noted at the end of this Sonnet distributed copy.

\section{References}

[1] J. C. Rautio, "A time-harmonic electromagnetic analysis of shielded microstrip circuits," Ph. D. dissertation, Syracuse University, Syracuse, NY, 1986.

[2] J. C. Rautio and R. F. Harrington, "An electromagnetic time-harmonic analysis of shielded microstrip circuits," IEEE Tran. Microwave Theory Tech., Vol. 35, No. 8, pp. 726-730, Aug. 1987.

[3] http://www.sonnetsoftware.com.

[4] J. C. Rautio, "A de-embedding algorithm for electromagnetics," International Journal of Microwave \& Millimeter-Wave Computer-Aided Engineering, Vol.1, No. 3, pp. 282-287, July 1991.

[5] J. C. Rautio, and V. I. Okhmatovski, "Unification of Double-Delay and SOC Electromagnetic Deembedding," IEEE Tran. Microwave Theory Tech., Vol. 53, No. 9, pp 2892-2898, Sep. 2005.

[6] J. C. Rautio, "De-embedding the effect of a local ground plane in electromagnetic analysis," IEEE Tran. Microwave Theory Tech, Vol. 53, No. 2, pp. 770-776, Feb. 2005.

[7] J. C. Rautio, "Electromagnetic component based design of planar circuits," IEEE Microwave Magazine, to be published, Aug. 2007.

[8] J. C. Rautio, "Synthesis of compact lumped models from electromagnetic analysis results," IEEE Tran. Microwave Theory Tech, submitted for publication.

[9] H. T. Hsu and J. C. Rautio, "Novel planar wideband omni-directional quasi log-periodic antenna," APMC 2005, Asia-Pacific Conference Proceedings, Vol. 4, 4-7 Dec. 2005.

[10] "Sonnet User's Guide," included with SonnetLite download at http://www.sonnetsoftware.com, pp. 281282.

Correction to the published paper: In the text it is suggested that the ripple in the minimum value of $\mathrm{S} 11$ is due to a surface wave coupled to the sidewall by the thin substrate supporting the antenna. Further numerical experiments indicate that the same ripple still occurs even when the substrate is limited to the immediate area around the dipole. The larger box size of Figure 5 still corrects the situation. Thus, the ripple has nothing to do with any surface wave. Our thanks to Dr. Hiroaki Kogure for this information. -- JCR 15 Nov 2007 